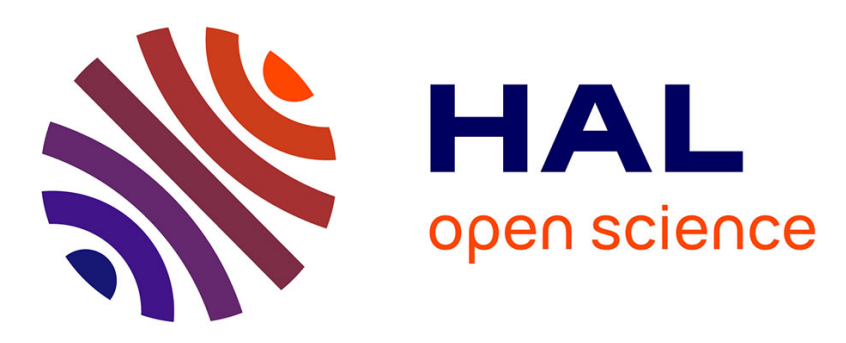

\title{
Efficient time domain HF geolocation using multiple distributed receivers
}

Ankit Jain, Pascal Pagani, Rolland Fleury, Michel Ney, Patrice Pajusco

\section{To cite this version:}

Ankit Jain, Pascal Pagani, Rolland Fleury, Michel Ney, Patrice Pajusco. Efficient time domain HF geolocation using multiple distributed receivers. EUCAP 2017: 11th European conference on antennas and propagation, Mar 2017, Paris, France. pp.1852 - 1856, 10.23919/EuCAP.2017.7928069 . hal01574834

\section{HAL Id: hal-01574834 \\ https://hal.science/hal-01574834}

Submitted on 6 Jul 2018

HAL is a multi-disciplinary open access archive for the deposit and dissemination of scientific research documents, whether they are published or not. The documents may come from teaching and research institutions in France or abroad, or from public or private research centers.
L'archive ouverte pluridisciplinaire HAL, est destinée au dépôt et à la diffusion de documents scientifiques de niveau recherche, publiés ou non, émanant des établissements d'enseignement et de recherche français ou étrangers, des laboratoires publics ou privés. 


\title{
Efficient Time Domain HF Geolocation Using Multiple Distributed Receivers
}

\author{
Ankit Jain ${ }^{1}$, Pascal Pagani ${ }^{1}$, Rolland Fleury ${ }^{1}$, Michel M. Ney ${ }^{1}$, Patrice Pajusco ${ }^{1}$ \\ ${ }^{1}$ Telecom Bretagne, Lab-STICC UMR CNRS 6285, Brest, France \\ ankit.jain@telecom-bretagne.eu
}

\begin{abstract}
This paper explores the effect of increasing number of receivers to evaluate the geographic location of the High Frequency (HF) transmitters for distances in the radius of $2000 \mathrm{~km}$. In the case of HF propagation over long distances, signals propagate using skywaves and are reflected back to the earth from the ionosphere. The Quasi-Parabolic (QP) model of the ionosphere provides analytic equations for ray path parameters through which the signal travel time is obtained. The position of the transmitter is found using time domain HF geolocation method namely Time Difference of Arrival (TDoA). An analysis of the QP model of the ionosphere is provided and limits of the model are emphasized. The geolocation algorithm for TDoA along with its mathematical equation is explained. Simulation results demonstrate that increasing the number of receivers leads to a significant improvement in the geolocation accuracy.
\end{abstract}

Index Terms-HF propagation, geolocation, ionosphere, multiple recivers, TDoA.

\section{INTRODUCTION}

HF radio waves can travel long distances using skywaves and therefore have their importance in military and civil applications. In such contexts, it is interesting to locate the position of the transmitter (Tx) by using the different types of information acquired from the signals received at the receiving station. Different methods are proposed to find the Direction of Arrival (DoA) of the signals by deploying a large array of co-located antennas [1]. Using time domain HF geolocation techniques such as Time of Arrival (ToA) and TDoA, signals received at different non co-located single antenna receivers (Rx's) are sufficient to locate the position of the Tx [2]. The ToA method requires a minimum of three Rx's to locate the position of the Tx [3]. Using the propagation path lengths obtained from the three Rx's, the Tx location can be retrieved using trilateration. If the transmission time of the signal is unknown, the Tx position can be retrieved by multilateration using TDoA at different Rx's. The TDoA method requires a minimum of four Rx's to locate the position of the Tx [4].

A long range $\mathrm{HF}$ radio transmission uses skywaves where the signal is reflected from the ionosphere. The signal reflected once from the ionosphere back to the earth can be referred as single hop mode. Using a single hop mode, transmissions using skywaves are possible up to distances of $3000 \mathrm{~km}$ at low elevation angles. In order to obtain the HF Tx location, it is necessary to obtain the group path lengths between the Tx and the Rx's which largely depend upon the electron density profile of the ionosphere. The skywaves are reflected back to the earth due to the presence of electrons in the ionosphere. The ionization in the ionosphere varies a lot with the solar cycle, geographical location, seasonally and diurnally. These ionospheric variations are the main issues in HF Tx geolocation through the skywaves. HF Tx geolocation can be further improved by the use of prediction models since it reduces time delay errors introduced by the ionosphere [5].

In our previous study of ToA geolocation [6], the Tx location was obtained without the knowledge of reflection height and the minimum number of 3 required $\mathrm{Rx}$ 's. The geolocation error was in the range of about 50-100 km depending on whether the transmitter was very close or far away from the Rx's. Another similar variant of HF geolocation was explained later in [7] based on TDoA and DoA methods which use multipath and achieve good geolocation performance without any ionospheric information. However, there is a requirement of antenna array on each reception site; accurate TDoA measurements are difficult to obtain in realistic scenario.

This paper studies, the effect of increasing the number of Rx's on the performance of HF Tx geolocation in the radius of $2000 \mathrm{~km}$. HF communication in the span of $2000 \mathrm{~km}$ is possible using a single hop mode. Further, to take into account the variable density of electrons in the ionosphere, a QP model of the ionosphere [8] is used. This model provides realistic group path lengths from which corresponding time delays are evaluated. Section II in the paper explains different aspects of ionospheric propagation. Section III explains the geolocation algorithm for TDoA along with its mathematical formulations. Simulation results are presented in Section IV in order to assess the $\mathrm{Tx}$ geolocation performance with an increasing number of receivers. Lastly conclusions are drawn in Section V.

\section{IONOSPHERIC PROPAGATION:PHYSICS AND MODELING}

\section{A. Structure of the Ionosphere}

The ionosphere [9] is a region extending from a height of about $50 \mathrm{~km}$ to $2000 \mathrm{~km}$ over the surface of the Earth. The neutral atoms present in this region are ionized by extreme ultraviolet (EUV) light from the sun. It results in the formation of free electrons and positively charged ions. These free electrons formed by photo-ionization are mainly responsible for the reflection of $\mathrm{HF}$ signal from different 
layers of the ionosphere facilitating long distance transmission.

Due to the presence of different gases at different altitudes in the ionosphere, there exist ionospheric layers. They are known as the D, E, $F_{1}$ and $F_{2}$ layer in the order of increasing altitude. The $\mathrm{F}_{2}$ layer is sometimes just referred as the $\mathrm{F}$ layer. Due to the opposing behavior between the density of the neutral atmosphere and the intensity of EUV radiation from the sun, the electron density of a given ionospheric layer is a convex function of the altitude, first increasing from zero to a maximum electron density, then again decreasing down to zero. The electron density is the least in the $\mathrm{D}$ layer and the maximum in the $\mathrm{F}$ layer.

The D layer exists at an altitude of about $50-100 \mathrm{~km}$ and is mainly responsible for the attenuation of the radio waves. The absorption or the attenuation of the signal reduces as the frequency of the signal is increased. The E layer exists from 100 to $150 \mathrm{~km}$ and can reflect HF signals. In the case of HF radio propagation, the $\mathrm{F}$ layer is the most important region. The height range for the $\mathrm{F}$ region is the highest which allows large communication paths. Furthermore, the electron density being the highest in the $\mathrm{F}$ region, it allows the reflection of the highest frequencies in the HF range.

\section{B. Analysis of the QP model}

To take account for the electron density profile for the radio signals propagating through the ionosphere, the QP model can be used. Developed by Croft and Hoogasian [8], it is obtained by a slight modification of the parabolic model used to define the electron density profile of the ionosphere. The QP model provides analytic expressions describing ray path parameters of oblique propagation considering the ionospheric medium. Using the QP model, the ground distance and group path distance can be evaluated between different Tx-Rx's pair for a specific elevation angle $(\beta)$ from the Tx and operating frequency $(f)$ by the formulae provided in [8]. The group path is obtained by multiplying the signal transmission time with the speed of light.

The electron density profile of a layer in the QP model is defined as follows:

$$
N_{e}=\left\{\begin{array}{c}
N_{m}\left(1-\left(\frac{r-r_{m}}{y_{m}}\right)^{2}\left(\frac{r_{b}}{r}\right)^{2}\right), \quad r_{b} \leq r \leq r_{m}\left(\frac{r_{b}}{r_{b}-y_{m}}\right) \\
0,
\end{array}\right.
$$

where $N_{e}$ is the electron density having a maximum value $N_{m}$ at a maximum distance of $r_{m}, r$ is the radial distance from the Earth's centre being equal to the sum of the Earth's radius and the height at which reflection occurs in the ionosphere, $r_{b}$ is the value of $r$ at the base of the layer and $y_{m}$ is the layer's semi-thickness [8].

The QP model of the ionosphere is a monolayer model providing ray path parameters considering reflection only from a specific layer in the ionosphere. It is a limitation of the QP model.

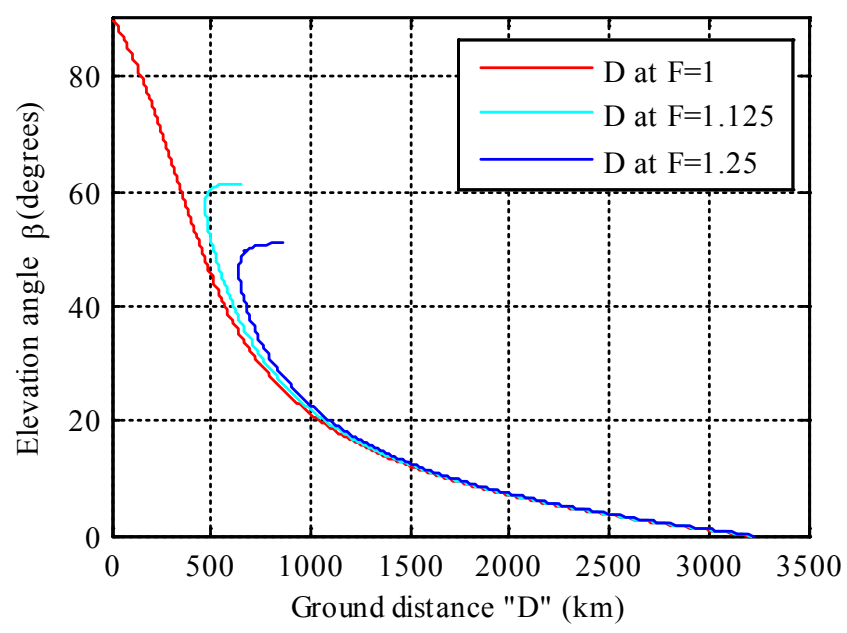

Fig. 1. Plot of ground distance using QP model at different values of F.

Fig. 1 represents the ground distance (D) plotted using QP model at different values of $F$, which is the ratio of the operating frequency $(f)$ of the source of HF radio waves to the critical frequency $\left(f_{c}\right)$ of the QP layer [8]. The maximum ground distance at different values of $F$ can be calculated at the elevation angle $\beta=0$ as seen in Fig. 1 . Thus, $D(\beta=0)$ is the maximum distance at which a Tx-Rx link could be established using single hop propagation through an ionospheric layer following the QP model.

The skip distance arises when $f_{c}$ of an ionospheric layer is greater than $f$ of the radio signal [10]. Hence, skip distance occurs only when the value of $F>1$. Skip distance can be found by calculating the minimum value of the ground distance curve for any specific value of $F>1$. Furthermore, it can also be found by computing the ground distance at which the slope of the curve is infinite for a plot of ground distance versus elevation angle. It is not possible to communicate using HF radio signals in the skip range using skywaves. The ground distance starts increasing again after reaching the skip distance for increasing elevation angle. It is due to high rays, also known as Pedersen rays, whereas the ground distance at which communication can be established before the skip distance range is due to low rays. Skip distance is a point where both the high and the low rays of a HF signal coincide.

\section{HF GEOLOCATION ALGORITHM}

A single hop transmission can be assumed for geolocation of HF transmitters in the radius of $2000 \mathrm{~km}$. The signal reaches the destination via a single reflection from the ionospheric layer. Fig. 2 represents the HF geolocation geometry considering a uniform, single layer ionosphere. The transmitter is located at point $T x$ and the receivers are located at point $R x 1$ and $R \times 2$. As per the Breit and Tuve's theorem [9], the group path between $R x 1-T x$ sketched by the red curve is equal to the triangular path $R x 1-I 1-T x$ and the same applies for $R x 2-T x$.

Due to the varying refractive index in the ionosphere, the signals propagate along a curved path. The apogee height of 


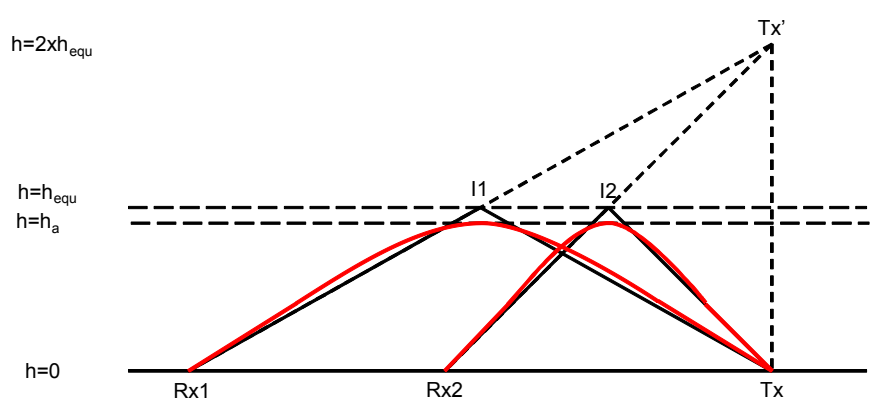

Fig. 2. Geometry of the HF localization principle considering uniform single layer ionosphere.

the group path length is denoted by $h_{a}$. An equivalent height $h_{\text {equ }}$ corresponding to the reflection height of the path length with the same group delay can be defined assuming the signals are reflected via specular reflection on a flat ionosphere. The ionosphere is assumed to be uniform, so the reflection height for all considered paths is similar for signals having similar elevation angles. The Earth is considered to be flat by neglecting its curvature.

Due to electromagnetic image theory, the length of the propagation path between $R x 1-T x$ is equal to $R x 1-I 1-T x$; similarly the propagation paths between $R x 2-T x$ is equal to $R x 2-I 2-T x^{\prime}$. The geolocation process consists of finding the coordinates of $T x^{\prime}$ which is situated at a height of $2 h_{e q u}$ vertically above the location of the $T x$ on a flat Earth. The coordinates of $T x^{\prime}$ can be found by multilateration in the case of TDoA (intersection of three hyperboloids) as explained in Section I.

The geometrical problem of identifying the intersection of three spheres or three hyperboloids requires solving systems of quadratic equations which cannot be solved analytically. A mathematical approach to obtain the location of $T x^{\prime}$ is explained further for the TDoA case using [11] as a reference.

\section{A. Mathematical approach of TDoA}

Let the receivers $\left(R x_{i}\right)$ be located at position $\mathbf{P}_{\mathbf{i}}=$ $\left(x_{i}, y_{i}, z_{i}\right)$ where $i=1,2,3 . . n$; let the transmitter $(T x)$ on the flat Earth surface be located at position $\mathbf{P}_{\mathbf{0}}=$ $\left(x_{0}, y_{0}, z_{0}\right)$. The coordinates of point $T x^{\prime}$ is located at $\mathbf{P}_{\mathbf{0}}^{\prime}=$ $\left(x_{0}, y_{0}, z_{0}^{\prime}\right)$ where $z_{0}^{\prime}$ is given by $(2)$ :

$$
z_{0}^{\prime}=z_{0}+2 h_{e q u}
$$

The propagation path length $D_{i}$ between a Tx-Rx pair via one hop mode is given by (3):

$$
D_{i}=c\left(t_{0}-t_{i}\right)=\left|\mathbf{P}_{\mathbf{0}}^{\prime}-\mathbf{P}_{\mathbf{i}}\right|
$$

where $t_{0}$ is the signal transmit time, $t_{i}$ is the signal arrival time at the $i^{\text {th }}$ receiver, $c$ is the speed of light, $D_{i}$ is the propagation path length between the transmitter and $i^{\text {th }}$ receiver and || represents the norm. Squaring (3), one obtains an equation which is given by:

$$
\left|\mathbf{P}_{\mathbf{0}}^{\prime}-\mathbf{P}_{\mathbf{i}}\right|^{2}=c^{2}\left(t_{0}-t_{i}\right)^{2}
$$

In the case of TDoA, the transmission time $t_{0}$ is unknown. Computing the difference between (3) for $i=$ $1\left(R x_{1}\right)$ and (3) for $i=2\left(R x_{2}\right)$; after further simplification one obtains (5) which is independent of $t_{0}$ and given by:

$$
\frac{-2\left(\mathbf{P}_{2}-\mathbf{P}_{\mathbf{1}}\right)^{\top} \cdot \mathbf{P}_{\mathbf{0}}^{\prime}}{t_{2}-t_{1}}+2 c^{2} t_{0}=\left(t_{1}+t_{2}\right) c^{2}-\frac{\left(\left|\mathbf{P}_{2}\right|^{2}-\left|\mathbf{P}_{\mathbf{1}}\right|^{2}\right)}{t_{2}-t_{1}}
$$

where $T$ represents the transpose operator.

As all receivers are situated on the Earth surface, one can consider $z_{1}=z_{2}=z_{3}=z_{0}$. After simplification, (5) has a form of linear equation with just 3 unknowns $\left(x_{0}, y_{0}, t_{0}\right)$ given by:

$$
\frac{-2\left(\mathbf{P}_{\mathbf{2}(x, y)}-\mathbf{P}_{\mathbf{1}(x, y)}\right)^{\top} \mathbf{P}_{\mathbf{0}(x, y)}^{\prime}}{t_{2}-t_{1}}+2 c^{2} t_{0}=\left(t_{1}+t_{2}\right) c^{2}-
$$

where $\mathbf{P}_{\mathbf{i}(x, y)}$ represents the projection of vector $\mathbf{P}_{\mathbf{i}}$ on the $(x, y)$ plane $(\mathrm{i}=1,2)$. In the TDoA method, time delays between different receivers are calculated considering a reference receiver. So with $3 \mathrm{Rx}$ 's, one can obtain two linear equations with 3 unknowns. Thus, to solve the equation for the 3 unknowns, a $4^{\text {th }} \mathrm{Rx}$ is used. Equation (6) is obtained using Rx's 1-2. Similarly using Rx pairs 1-3 and 1-4, respectively, one can obtain 2 more equations. Thus, a linear system with 3 independent equations with 3 unknowns is obtained having the following form:

$$
\left\{\begin{array}{l}
a_{11} x_{0}+a_{12} y_{0}+a_{13} t_{0}=b_{1} \\
a_{21} x_{0}+a_{22} y_{0}+a_{23} t_{0}=b_{2} \\
a_{31} x_{0}+a_{32} y_{0}+a_{33} t_{0}=b_{3}
\end{array}\right.
$$

The constants of $1^{\text {st }}$ row of (7) can be obtained by identification with (6) which yields:

$$
\begin{gathered}
a_{11}=\frac{-2\left(x_{2}-x_{1}\right)}{t_{2}-t_{1}} ; a_{12}=\frac{-2\left(y_{2}-y_{1}\right)}{t_{2}-t_{1}} ; a_{13}=2 c^{2} \\
b_{1}=c^{2}\left(t_{2}+t_{1}\right)-\frac{\left(x_{2}^{2}-x_{1}^{2}\right)}{t_{2}-t_{1}}-\frac{\left(y_{2}^{2}-y_{1}^{2}\right)}{t_{2}-t_{1}}
\end{gathered}
$$

Similarly from the other 2 equations obtained from $\mathrm{Rx}$ pairs 1-3 and 1-4, constants of other rows of (7) can be obtained. Finally, the transmitter coordinates $\left(x_{0}, y_{0}\right)$ and the transmit time $t_{0}$ can be obtained by doing a simple matrix inversion operation. The value of $z_{0}^{\prime}$ can be obtained by substituting $\left(x_{0}, y_{0}\right)$ in (4). Finally the equivalent height $h_{\text {equ }}$ can be obtained by resolving (2).

Note: Equation (7) can be realized in the form of $\left(x_{0}, y_{0}, t_{0}\right)^{\top}=\mathbf{A}^{-1} \mathbf{B}$ to obtain the transmitter coordinates. In the case of more receivers, the matrix inversion is done using the pseudo inverse of matrix A. 


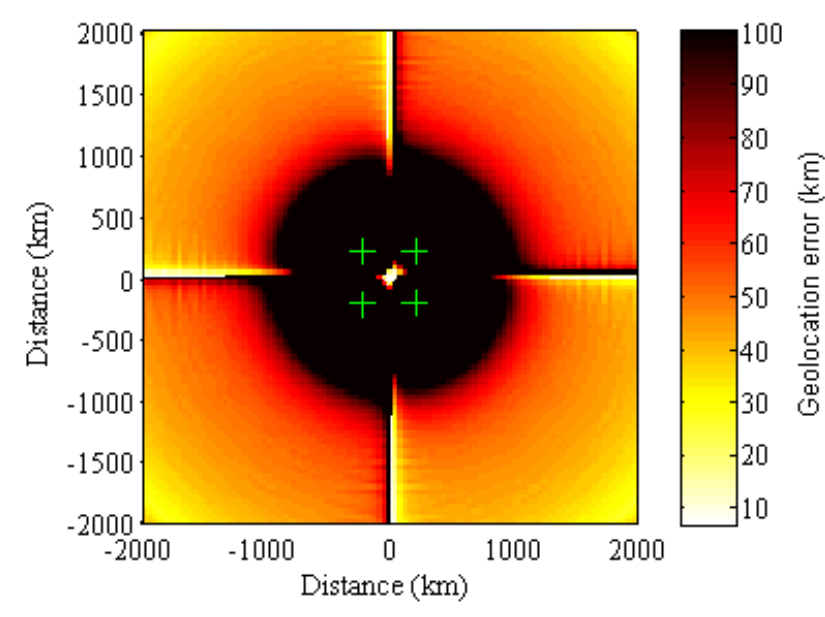

Fig. 3. Geolocation error using TDoA with $4 \mathrm{Rx}$ 's for a QP ionosphere.

\section{Simulation RESUlts}

\section{A. Quasi-Parabolic Ionosphere with a Fixed Height}

Fig. 3 represents geolocation error in locating Tx on a square grid with a length of $4000 \mathrm{~km}$ using the least number of 4 receivers required by the TDoA method. The 4 receivers are placed in a manner that they approximately form a square with the center at the origin of the square grid. The artifact seen along both the axes of the grid in Fig. 3 is due to the approximate symmetry formed due to the manner in which the receivers are placed. The length of the sides of the square formed by the receiver is approximately $420 \mathrm{~km}$. These four receivers are illustrated by green "+" symbols in Fig. 3. The group path lengths between different receivers and all possible transmitters on the grid are evaluated using the analytic expression of the QP model. The values used to define the QP model in all simulations are: $F=0.8$, $h_{m}=300 \mathrm{~km}$ and $y_{m}=100 \mathrm{~km}$. The coordinates of the transmitter can be found by the intersection of three hyperboloids using the geolocation algorithm of TDoA explained in Section III. The height of maximum ionization $\left(h_{m}\right)$ of the QP ionosphere is assumed to be fixed, thus leading to a uniform ionosphere. The geolocation algorithm being dependent on the reference receiver, the $\mathrm{Tx}$ coordinates are evaluated considering each receiver as the reference receiver. The final $\mathrm{Tx}$ location is obtained by computing the mean of the Tx coordinates obtained from each reference receiver. Finally, the geolocation error is evaluated by calculating the difference between the true location and the obtained final location. All the geolocation error greater than $100 \mathrm{~km}$ is represented by black color.

The observed geolocation error is very large in the case when the Tx site is located at a distance less than around 600 $\mathrm{km}$ from a $\mathrm{Rx}$ site. This geolocation error is higher due to large variation of the equivalent height between a Tx and different $\mathrm{Rx}$ configurations when they are placed close to each other. However, as a Tx site is further away from the origin, the geolocation error decreases. The Tx located at a distance range of around 1200 to $2000 \mathrm{~km}$ from the origin of

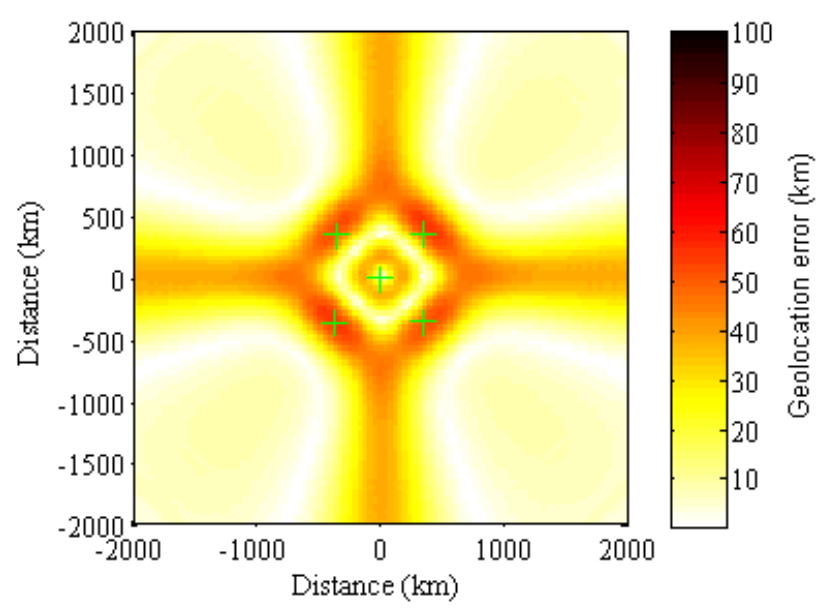

Fig. 4. Geolocation error using TDoA with $5 \mathrm{Rx}$ 's for a QP ionosphere.

the square grid could be located with an error of about 20-50 $\mathrm{km}$ using four receivers.

Fig. 4 represents geolocation error calculated for each $\mathrm{Tx}$ location using five receivers with the TDoA method. The 4 receivers are placed in a way that they approximately form a square with the length of side approximately equal to $710 \mathrm{~km}$ and the fifth receiver is placed at the centre of the square grid. The geolocation error for each Tx location, assuming refraction from a uniform QP ionosphere is calculated in a similar manner as done in the four receiver case. As seen in Fig. 4, the maximum geolocation error is around $50 \mathrm{~km}$ very close to the receivers. With the use of an additional receiver and placing it in an optimum position, the geolocation accuracy is improved.

Fig. 5 represents the CDF of geolocation error in locating transmitters on a square grid with a length of $4000 \mathrm{~km}$ using different configurations of the number of receivers which are placed approximately uniformly over the grid. It is also assumed that the refraction of radio signals is from a QP ionosphere with fixed height. The numbers in the legend box of Fig. 5 corresponds to the number of receivers. From Fig. 5 , it is also evident that $80 \%$ of the geolocation error is less than $95 \mathrm{~km}$ using four receivers whereas $80 \%$ of the geolocation error is less than $30 \mathrm{~km}$ using five receivers. With five receivers, the median of the geolocation error is reduced by almost $85 \%$ compared to the median of the geolocation error with four receivers. This gain in terms of reduced geolocation error is quite high at the expense of just one additional receiver. Furthermore, with increasing the number of receivers the geolocation error keeps decreasing but the improvement is less significant. As seen from Fig. 5, there is only a slight improvement in the geolocation error when the number of receivers is increased from 13 (yellow curve) to 17 (cyan curve). From Fig. 5, it is also evident that $93 \%$ of the geolocation error is less than $10 \mathrm{~km}$ and the maximum geolocation error is just $25 \mathrm{~km}$ using $17 \mathrm{Rx}$ 's. Note that the Tx coordinates is evaluated using only the receivers for which the transmitter lies within their radio horizon. 


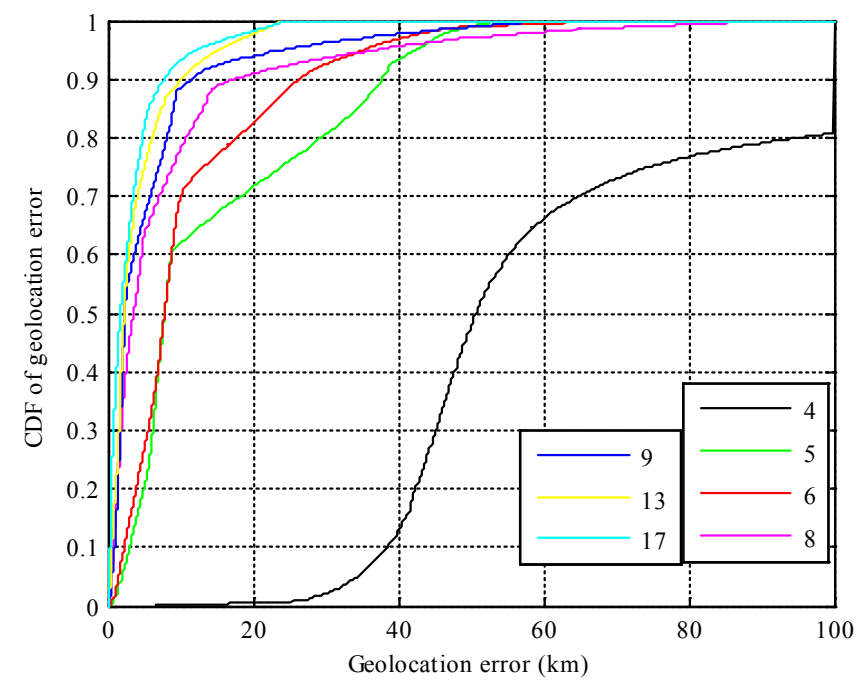

Fig. 5. CDF of geolocation error for different numbers of Rxs assuming a QP ionosphere with fixed height.

\section{B. Quasi-Parabolic Ionosphere with a Variable Height}

In order to obtain the location of the Tx using more realistic conditions of the ionosphere, the height of maximum ionization $h_{m}$ is varied as a Gaussian distribution with a mean of $300 \mathrm{~km}$ and variance of $20 \mathrm{~km}$ all across the square grid of length $4000 \mathrm{~km}$. Fig. 6 represents the CDF of geolocation error in locating transmitters on a square grid with a length of $4000 \mathrm{~km}$ with different configurations of the number of receivers and the height $h_{m}$ being varied as a Gaussian distribution. The numbers in the legend box of Fig. 6 corresponds to the number of receivers. As expected, the geolocation error is larger in this case for a similar number of receivers when compared to refraction from a uniform QP ionosphere. From Fig. 6, it is seen that around $20 \%$ of the geolocation error are less than $100 \mathrm{~km}$ using four receivers whereas around $75 \%$ of the geolocation error are less than $100 \mathrm{~km}$ using five receivers. The geolocation accuracy improves considerably when the number of receivers is increased from 5 to 9 and then, from 9 to 13 as shown in Fig. 6.The geolocation error keeps decreasing with increasing number of receivers.

\section{CONCLUSIONS}

This paper presents, HF geolocation using TDoA with variable number of receivers. These techniques make use of single antenna receivers which are a cost effective solution and can be easily deployed. The group path between a Tx-Rx pair for oblique propagation is obtained using the QP model. Furthermore, the mathematical approach for geolocation is also explained for the TDoA case. Simulation studies show that the geolocation error is significantly reduced by increasing the number of receivers.

$95 \%$ of the geolocation error is approximately less than 10 $\mathrm{km}$ using $17 \mathrm{Rx}$ 's for a uniform QP ionosphere; $95 \%$ of the geolocation error is less than $40 \mathrm{~km}$ using $17 \mathrm{Rx}$ 's for a QP ionosphere having a variable height. The position of the receivers also plays an important role in the geolocation of

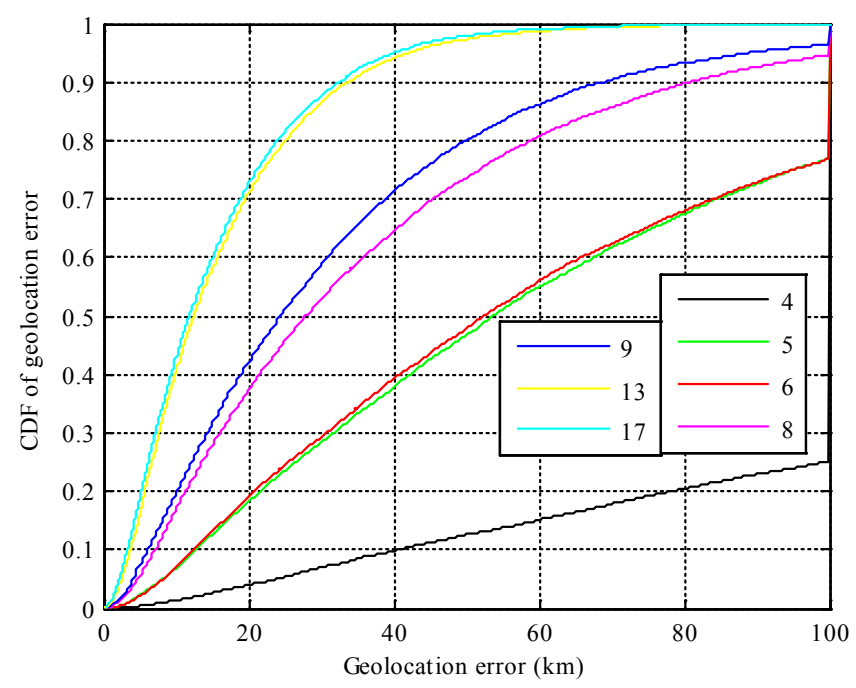

Fig. 6. CDF of geolocation error for different numbers of Rxs assuming a $\mathrm{QP}$ ionosphere with variable height following a Gaussian distribution.

the Tx. Several aspects related to the position of the receivers will be investigated in our future research. An experiment will be setup in the near future with four or five receivers to confirm these simulation results.

\section{ACKNOWLEDGMENT}

Part of this study has been funded by the Carnot Institute and the Brittany Region of France.

\section{REFERENCES}

[1] P. J. D. Gething, "HF direction finding," Proceedings of the Institution of Electrical Engineers, vol. 113, no. 1, pp. 49-61, 1966.

[2] P. Pagani, I.E.Mejri, et al." "Time domain HF geolocation: Experimental measurements and preliminary results," in 10th European Conference on Antennas and Propagation (EuCAP), 2016.

[3] A. Bensky, "Wireless positioning technologies and applications," Artech House, pp. 161-188, n ISBN-13: 978-1-59693-130-5.

[4] D. J. Torrieri, "Statistical theory of passive location systems," IEEE Trans. on Aerospace and Electronic Systems, vol. AES-20, no. 2, pp. 183-198, Mar. 1984.

[5] N. N. Rao, R. I. Beckwith, and E. W. Ernst, "Prediction of differential time-delay errors in HF hyperbolic position-fixing systems," IEEE Transactions on Aerospace and Electronic Systems, vol. AES-10, no. 6, pp. 765-769, 1974.

[6] P. Pagani, R. Fleury, et al., "A study of HF transmitter geolocation through single-hop ionospheric propagation", in European Conference on Antennas and Propagation, 2014, pp. 2689-2693.

[7] G. Wang, X. Jiang, S. G. Razul, C. M. See, and Z. Lin, "Passive TDOA and DOA based HF geolocation without ionosphere information," in 10th International Conference on Information, Communications and Signal Processing (ICICS), 2015.

[8] T. A. Croft and H. Hoogasian, "Exact ray calculations in a quasiparabolic ionosphere with no magnetic field", Radio Science, vol. 3, no. 1, pp. 69-74, 1968.

[9] K. Davies, "Ionospheric radio," IEE Electromagnetic Waves Series 31, pp. 124-158, n ISBN : 086341186 X, May 1989.

[10] G.S. Sales, High Frequency (HF) Radiowave Propagation. PL-TR-922123, Philips Laboratory, Hanscom AFB, MA, April 1992.

[11] Bakhoum, E.G., "Closed-form solution of hyperbolic geolocation equations," in IEEE Transactions on Aerospace and Electronic Systems, vol.42, no.4, pp.1396-1404, Oct. 2006. 